ARTICLE

https://doi.org/10.1057/s41599-019-0241-x

\title{
Interaction effects between consumer information and firms' decision rules in a duopoly: how cognitive features can impact market dynamics
}

\author{
Aurélien Nioche (10) 1,2,3,4,5, Basile Garcia'2,3,4,5,6,7,8, Thomas Boraud ${ }^{4,5,9}$, Nicolas Rougier ${ }^{4,5,8,10}$ \& \\ Sacha Bourgeois-Gironde $2,3,11$
}

\begin{abstract}
Duopolies are situations where two independent sellers compete for capturing market share. Such duopolies exist in the world economy (e.g., Boeing/Airbus, Samsung/ Apple, Visa/MasterCard) and have been studied extensively in the literature using theoretical models. Among these models, the spatial model of Hotelling (1929) is certainly the most prolific and has generated subsequent literature, each work introducing some variation leading to different conclusions. However, most models assume consumers have unlimited access to information (perfect information hypothesis) and to be rational. Here, we consider a situation where consumers have limited access to information and explore how this factor influences the behavior of competing firms. We first characterized three decision-making processes followed by individual firms (maximizing one's profit, maximizing one's relative profit with respect to the competitor; or tacit collusion) using a simulated model, varying the level of information of consumers. These manipulations alternatively lead the firms to minimally or maximally differentiate their relative position. We then tested the model with human participants in the role of firms and characterized their behavior according to the model. Our results demonstrate that limited access to information by consumers can actually induce a mutually beneficial non-competitive behavior of firms, which is not traceable to explicit collusive strategies. Imperfect information on the part of consumers can hence be exploited by firms through basic and blind decision rules.
\end{abstract}

\footnotetext{
${ }^{1}$ Aalto University, School of Electrical Engineering, Department of Communications and Networking, 02150 Espoo, Finland. ${ }^{2}$ Institut Jean Nicod, Département d'Etudes Cognitives, ENS, EHESS, PSL Research University, 75005 Paris, France. ${ }^{3}$ Institut Jean Nicod, Département d'Etudes Cognitives, CNRS, UMR 8129, 75005 Paris, France. ${ }^{4}$ Institut des Maladies Neurodégénératives, Université de Bordeaux, 33000 Bordeaux, France. ${ }^{5}$ Institut des Maladies Neurodégénératives, CNRS, UMR 5293, 33000 Bordeaux, France. ${ }^{6}$ Laboratoire de Neurosciences Cognitives Computationnelles, Département d'Etudes Cognitives, ENS, PSL Research University, 75005 Paris, France. ${ }^{7}$ Laboratoire de Neurosciences Cognitives Computationnelles, INSERM, U960, 75005 Paris, France. ${ }^{8}$ Inria Bordeaux Sud-Ouest, 33405 Talence, France. ${ }^{9}$ Centre Expert Parkinson, CHU Bordeaux, 33000 Bordeaux, France. ${ }^{10}$ LaBRI, Université de Bordeaux, INP, CNRS, UMR 5800, 33405 Talence, France. ${ }^{11}$ Laboratoire d'Economie Mathématique et de Microéconomie Appliquée, Université Panthéon Assas, 75006 Paris, France. These authors contributed equally: Aurélien Nioche, Basile Garcia. These authors jointly supervised this work: Nicolas P. Rougier, Sacha Bourgeois-Gironde Correspondence and requests for materials should be addressed to A.N. (email: nioche.aurelien@gmail.com)
} 


\section{Introduction}

uopolies are situations where two independent sellers compete for capturing market share. There are actually numerous examples of such situations in the world economy (e.g., Boeing/Airbus, Samsung/Apple, Visa/MasterCard). What is particularly interesting in these situations is the fact that it is not rare to observe sellers adopting a similar positioning on the market, both in geographical terms and in terms of product differentiation (e.g., Burger King/McDonalds). This can appear counterintuitive at first glance: one could spontaneously assume that sellers would try to avoid such behavior in order to reduce competition. The first formal model proposed by Hotelling (1929) for describing such situations has provided an explanation on why and how firms could be incentivized to minimally differentiate. His model considers a pool of consumers that are uniformly spread over a one-dimensional segment. Two firms selling the same product have to decide where to locate on this segment and what price to offer for their product, knowing that each consumer will choose a firm according to its relative distance (linear transportation costs) and the price of the product. The original study holds that in such conditions, firms tend to aggregate and compete near the center of the segment (minimal differentiation principle) due to the effort of the firms to capture the largest number of consumers. However, subsequent research (d'Aspremont et al., 1979; Cremer et al., 1991; Economides, 1993; Brenner, 2005) demonstrated the existence of an antagonist principle of maximal differentiation, using either quadratic transportation costs, a higher number of competitors or a higher number of dimensions on which firms can differentiate themselves. In the end, both minimal and maximal differentiation can be incentivized and observed (Irmen and Jacques-François, 1998). Here, we show how the level of information of consumers may induce different behavior for the two firms, depending on their strategies.

Several experimental studies have already attempted to characterize the various factors influencing differentiation. For instance, Kruse et al. (2000) allowed for communication between participants in the role of firms and showed that they tend to group in the center when communication is limited, but on the contrary, to differentiate themselves if communication is unlimited (cooperation). Similarly, Kephart and Friedman (2015) setup a protocol contrasting continuous and discrete time and demonstrated that continuous time could trigger a maximal differentiation strategy, as it allows some form of communication, and as a consequence, some form of cooperation. These two findings brought together suggest that quick and/or full information transmission can help to reach a cooperative equilibrium in a typical Hotelling's model. Several other studies brought up arguments supporting the robustness of the minimal differentiation phenomenon such as, for example, the four-player version of the game by Huck et al. (2002) or in BarredaTarrazona et al. (2011), where subjects tended to group in the center under several experimental conditions. Although there is a treatment in Barreda-Tarrazona et al. (2011) with human subjects as consumers, what is common to all these works is their shared assumption of the fact that firms are competing to capture rational and fully informed consumers even when they document spatial behavior that departs from Nash equilibrium when it theoretically exists. The case when consumers have no full informational access to the firms' strategies and when firms must compete over this less than completely informed consumers have not been addressed, to our knowledge, in the experimental literature related to Hotelling (1929). It has yet important implications as it is a common fact that consumers are not fully aware of all options available on the markets they participate in and that firms know and anticipate this fact in their own strategies.
Stigler (1961) argued that the information question should be fully taken into account in such competition models, as it can deeply impact the nature of equilibria. This is particularly important as consumer choices are known to be subject to several biases and based on partial information (Thaler, 1980; Kahneman, 2003). More precisely, the uncertainty resulting from the imperfect nature of information has been shown to provide an incentive for the firms to regroup and transparency of the market is, thus, a prominent factor for differentiation (Webber, 1972; Stahl, 1982). In line with predictions from earlier studies (Eaton and Richard, 1975; Brown, 1989; Dudey, 1990; Schultz, 2009), we postulate that in a duopoly context, the consumers' access to information is a critical factor for the differentiation of the two firms.

We thus defined a formal turn-based model (Prescott and Vissher, 1977; Loertscher and Muehlheusser, 2011) that allows us to explicitly manipulate the amount of information available to consumers while retaining their rational nature. Agents can act rationally under partial information and thereby induce observable organizational patterns in the market that differ from what is expected under perfect information. We test the hypothesis that the amount of information accessible to consumers can variably drive the differentiation of the two firms: when this amount is low, firms will be maximally differentiated; when this amount is high, firms will be minimally differentiated. We test this hypothesis using a simulation where we consider three decisionmaking processes for the firms, namely (i) a maximization of short-term profits, (ii) a maximization of the difference of profits between the firm and its opponent, (iii) a maximization of the profits of the two firms. Following Rubinstein's prescriptions (Rubinstein, 1991), the aim of these decision rules is to incorporate the potential perception of the situation by the decision makers. These rules indeed constitute plausible behavioral responses on the part of firms in the light of partial information on the part of consumers. These decision rules helped us to characterize the behavior of human subjects for the experimental part of this work where subjects play the role of the firms under different informational conditions. The choice of using the first decision rule is straightforward: a firm only pays attention to its current own profit, considering further expectations about the future not reliable. A firm may consider the behavior the other firm either too difficult to compute or not reliable. This is tantamount to ignore the other player strategies. This type of "blind" decision rule has a presence in the Industrial Organization literature stemming as far as Rothschild (1947) in which securing one's profit is the only rule by which a firm's behavior is guided.

Regarding the second decision rule, motivations are dual. It could lie on an anchoring bias (Tversky and Kahneman, 1974): it is difficult to evaluate the success of a move per se, a move is considered efficient if it leads to better profits than its opponent. In other words, firms' strategies evaluation relies on comparisons to a given point instead of evaluation in absolute terms. Secondly, it could be due to a zero-sum bias (Meegan, 2010; Różycka-Tran et al.,), even if in our model, the profit of one firm is not necessarily made at the expense of the other firm (see 'Methods' section). Indeed, considering-sometimes wrongfully-that a greater profit for its opponent is a profit loss for itself, a firm could decide to make its choice only considering the profit difference.

The use of the third heuristic lies on the expectation of tacit collusion (TC) with the opponent: if both firms try to maximize their own profit as well as the profit of their opponent, it would avoid the drawbacks of a competitive situation and leads to higher profits. It could be also seen as a search for Pareto optimality (Pareto, 1964), that is to say following the strategies that lead to a 
repartition of profits such as no firm could earn more, otherwise, it would be at the expense of the other.

Therefore, we hypothesized that (i) depending on the information available to consumers, either a minimum differentiation or a maximal differentiation can apply, (ii) the effect of the information available to consumers can be modulated by the firms' decision rules.

\section{Methods}

Model description. We consider a unidimensional normalized space $X$ discretized into $n_{\text {cons }}$ evenly spread locations such that $x_{i}$ $=(i-1) /\left(n_{\text {cons }}-1\right)$. We consider a set of $1+p_{\max }-p_{\min }$ integer prices $P$ ranging from $p_{\min }$ to $p_{\max }$. We consider two firms $\left\{F_{j}\right\}_{j \in[1,2]}$ and a group of $n_{\text {cons }}$ consumers $\left\{C_{i}\right\}_{i \in\left\{1, \ldots, n_{\text {cons }}\right\}}$.

Each firm $F_{i}=\left(x_{i}, p_{i}\right)$ is characterized by a position $x_{i}$ and a price $p_{i}$. Consumers are uniformly spread over space such that $x_{i}$ $=(i-1) /\left(n_{\text {cons }}-1\right)$. View radius is defined on a per-experiment basis and is the same for all the consumers during an experiment. The firm position is a free variable and must correspond to a consumer position such that there are only $n_{\text {cons }}$ different possible positions for a firm. Price $p_{\mathrm{i}}$ is a free variable and is discrete: there are $P$ possible prices spread uniformly between a minimal price $p_{\text {min }}$ and a maximal price $p_{\text {max }}$. Simulations are turn-based (Prescott and Vissher, 1977; Loertscher and Muehlheusser, 2011). We distinguish at each turn an active firm that is allowed to select a strategy and a passive firm that has to wait for the next turn to react and deploy its own strategy. More specifically, at turn $t$, Firm A $\left(F_{1}\right.$ or $\left.F_{2}\right)$ chooses its location and its price, consumers choose a firm and profits are collected for both firms. At turn $t+$ 1, Firm B $\left(F_{2}\right.$ or $\left.F_{1}\right)$ chooses its location and its price (while Firm A keeps location and price from turn $t$ ), consumers choose a firm and profits are collected for both firms. Let $\Pi_{i}$ be the profit of the firm $F_{i}$ for a single turn defined by $\Pi_{i}=p_{i} \cdot q_{i}$ with $p_{i}$ the price at which $F_{i}$ sells its product, and $q_{i}$ the quantity $F_{i}$ sold. There is no production cost. Consumers are able to buy only one product per turn. They consume it immediately, in such a manner that they do not constitute any stock. This implies that a firm produces a maximum of $n_{\text {cons }}$ products per turn.

Each consumer $C_{i}=\left(x_{i}, r_{i}\right)$ is characterized by a position $x_{i}$ and a view radius $r_{i}$. The view radius defines a segment centered on the consumer $\left[x_{i}-r_{i}, x_{i}+r_{i}\right]$. Only firms located inside this segment are considered by the consumer (see Fig. 1). Consequently, at each turn, some consumers will see only one firm and will be captive since they cannot choose what firm to buy from. Some consumers will see both firms and are named volatile because they can choose any of the two firms depending on their choice criterion. Some consumers won't see any firms and cannot buy, and, thus, are named ghost consumers. A view radius of 0 means the firm has to be at the same position to be seen while a radius of 1 means the firm is seen by all the consumers. Reciprocally, and depending on the consumer view radius, firms have access only to a subset of all the consumers, they are named the potential consumers and represent the sum of captive and volatile consumers.

Parameters. For all the simulations, we used the following parameters: $n_{\text {cons }}$ (number of consumers) $=21, n_{\text {price }}$ (number of prices $)=11, \quad p_{\min } \quad($ minimal price $)=1, \quad p_{\max } \quad$ (maximal price) $=10, n_{\text {turn }}=100$. The initial position and price for the passive firm (first turn) are randomly assigned. The view radius $(r)$ is the same for all the consumers and is comprised between 0 and 1 . For each of the three different decision-making processes, we ran (i) 1000 simulations with $r$ randomly (uniformly) drawn between 0 and 1 for each simulation; (ii) 64 additional simulations with $r=0.25$ and $r=0.50$ respectively, in order to characterize experimental data.

Decision-making processes. Consumers do not choose the amount of information they dispose of. They may see zero, one or two firms. In the event that they do not see any firm, they are unable to buy and have to wait for the next turn. If they see a single firm, they have no means to compare prices and have to buy from this firm, independently of the price (each consumer has an unlimited budget). When they are able to see the two firms, they buy from the firm offering the lowest price. In the specific case where prices of both firms are equal, they choose randomly between the two. Firms have perfect knowledge of the environment: they know (i) the price and the position of the opponent, (ii) the location of each consumer $x_{\mathrm{i}}$, (iii) the view radius $r_{i}$ of each consumer and (iv) the decision-making method of consumers. Firms from two different simulations can differ in their decision-making process but two firms from the same simulation share the same decision-making process. Decisionmaking process of a firm is either one of the three following decision rules:

Profit maximization (PM). Each time an active firm plays, it computes the potential profits for all the possible position-price couples regarding the current decision-making process of the passive firm, and chooses the couple position-price that maximizes profit (in the case where several couples position-price lead to the same best payoff, the firm chooses randomly between these moves);

Difference maximization (DM). If Firm $A$ is the active firm, the difference between its own profit and the profit of Firm B is computed for each possible move, and the move leading to the greatest difference is chosen (in case of multiple moves leading to the greatest difference, the move is randomly chosen between those moves);

Tacit collusion (TC). The distance to the maximum profit for Firm $\mathrm{A}$ and the distance to the maximum profit for Firm B are computed for each move the active firm can play, the chosen move is the one leading to the minimum sum of the distances.

Firm decision-making process. The choice space is defined by the set $Y$, that is the Cartesian product of all possible locationprice couples:

$$
Y=\left\{\left(x_{i}, p_{j}\right)\right\}_{x_{i} \in X, p_{j} \in P}
$$

The expected profit of the firm A is defined by the number of potential consumers (the sum of captive and volatile consumers) that a firm can expect when making the move $y_{\alpha}$, that is locating at position $x_{i}$ and setting price $p_{j}$.

Let the boolean-valued function $V_{C k}$ determine if a consumer $C_{k}$ sees the location $x_{i}$ :

$$
V_{C_{k}}\left(x_{i}\right)=\left\{\begin{array}{l}
1 \text { if } x_{k}-r_{k} \leq x_{i} \leq x_{k}+r_{k} \\
0 \quad \text { otherwise }
\end{array}\right.
$$

Let the function $V_{C k}$ define the profit that the firm A can expect from a consumer $C_{k}$ for the move $y_{\alpha}=\left(x_{i}, p_{j}\right)$, knowing that the 


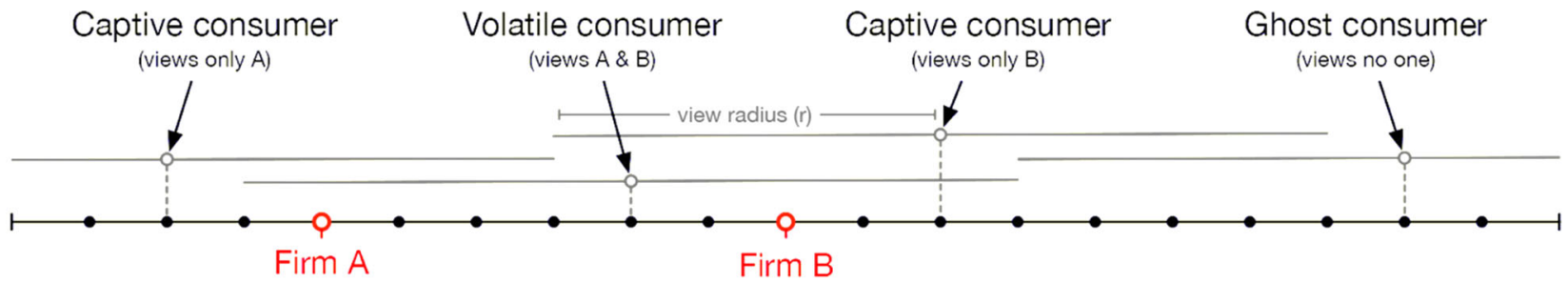

Low level of information $(r=0.25)$

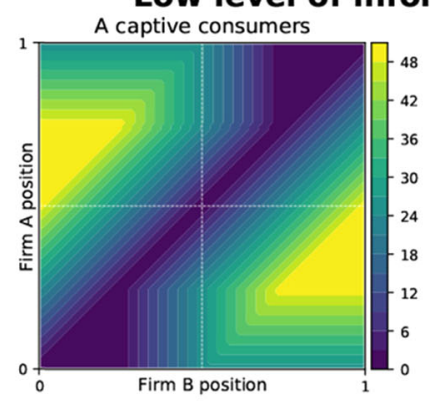

Volatile consumers
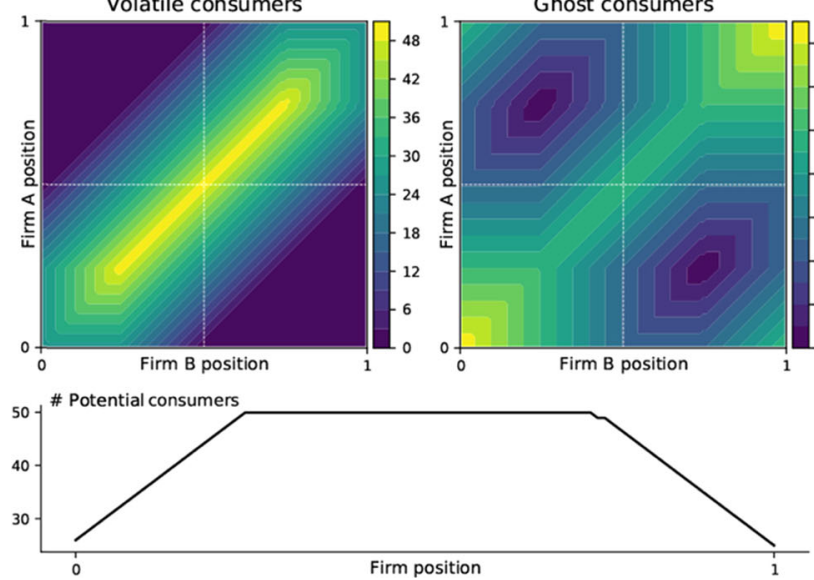

Firm B position Ghost consumers
High level of information $(r=0.50)$
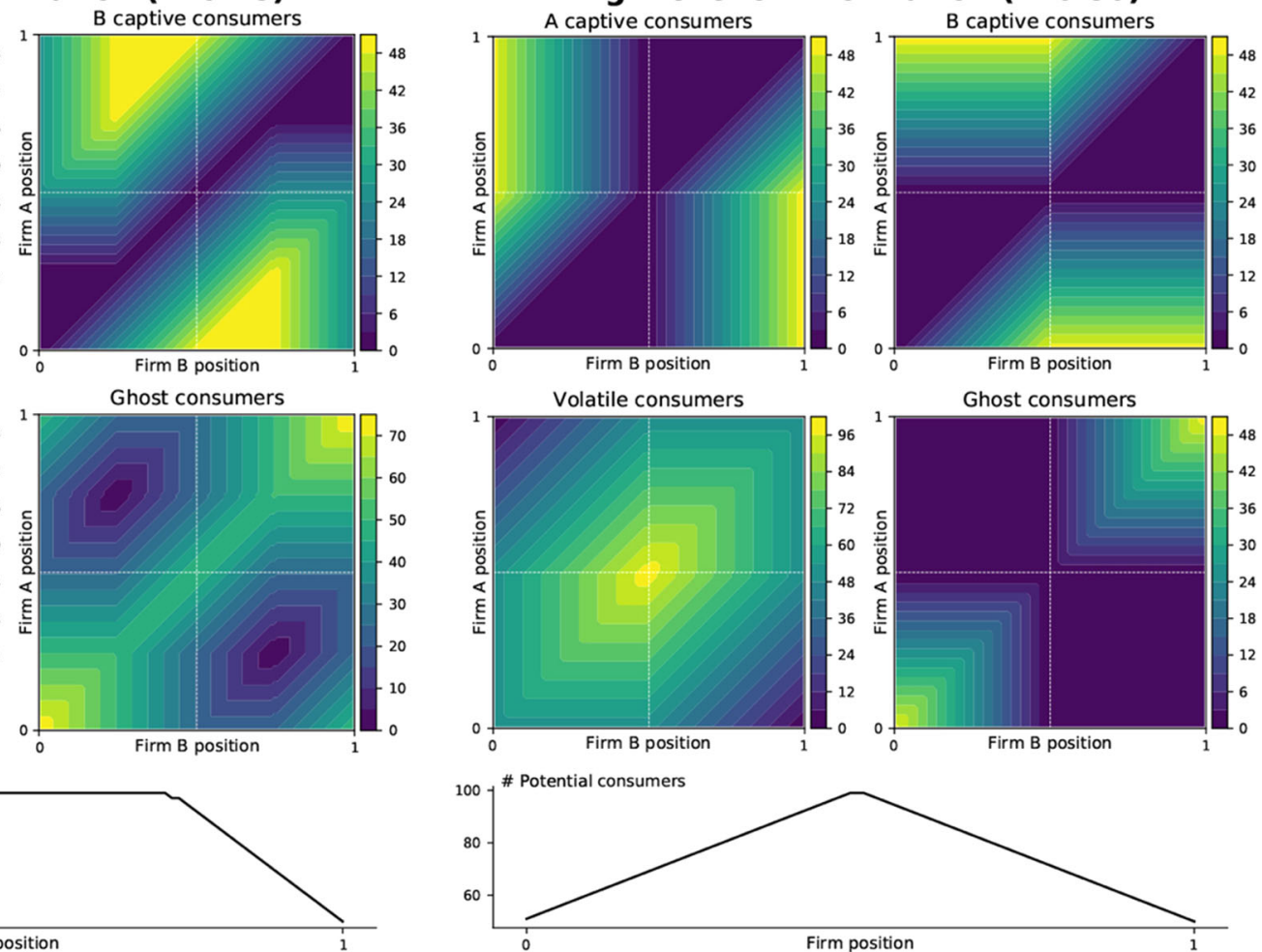

Fig. 1 Model. The model is represented by a one-dimensional line segment over which consumers (black dots) are spread uniformly. Firms (outlined red dots) are free to position themselves on any consumer position. Consumers view firms that fall within their view radius such that some consumers view only one firm (captive consumers), some consumers view both firms (volatile consumers) and some consumers view none (ghost consumers). The number of captive, volatile, and ghost consumers for a firm depends on the size of the view radius of the consumers and the respective position of the two firms. The number of captive consumers increases as the view radius of consumers decreases. The number of potential consumers (captive + volatile) of a firm is a function of the position of the firm and the view radius of the consumers. When the level of information is high $(r=0.50)$, there is a unique position where all the consumers are potential consumers for a firm. When the level of information is low $(r=0.25)$, there is a whole segment where exactly half the consumers are potential consumers for a firm

move of the opponent is $y_{\beta}=\left(x_{m}, p_{n}\right)$ :

$W_{C_{k}}^{A}\left(y_{\alpha}, y_{\beta}\right)=\left\{\begin{array}{cc}0 & \text { if }\left[p_{j}>p_{n} \wedge V_{C_{k}}\left(x_{m}\right)=1\right] \vee V_{C_{k}}\left(x_{i}\right)=0, \\ 0.5 & \text { if } p_{j}=p_{n} \wedge V_{C_{k}}\left(x_{m}\right)=1, \\ 1 & \text { otherwise. }\end{array}\right.$

Then, the expected profit of the firm A for a given move $y_{\alpha}$ is obtained from the function $E^{A}$, knowing that the move of firm $\mathrm{B}$ is $y_{\beta}$ :

$$
E^{A}\left(y_{\alpha}, y_{\beta}\right)=\sum_{k=1}^{n_{\text {cons }}} W_{c k}^{A}\left(y_{\alpha}, y_{\beta}\right)
$$

A firm makes the move $y_{\lambda}$ (that is a specific combination of position and price) using one of the following decision rules: profit maximization (PM), difference maximization (DM), or tacit collusion (TC).
When following a PM decision rule, the active firm $\mathrm{A}$ maximizes its expected profit $E_{y_{i}, y_{\beta}}^{A}$ such that:

$$
\lambda=\underset{i}{\arg \max }\left(\left\{E^{A}\left(y_{i}, y_{\beta}\right)\right\}_{y_{i} \in Y}\right)
$$

When following a DM decision rule, the active firm A maximizes the difference between its own expected profit and the corresponding expected profit of the passive firm B:

$$
\lambda=\underset{i}{\arg \max }\left(\left\{E^{A}\left(y_{i}, y_{\beta}\right)-E^{B}\left(y_{i}, y_{\beta}\right)\right\}_{y_{i} \in Y}\right)
$$

When following a TC decision rule, the firm A makes the move maximizing both its own expected profit and the expected profit of its opponent, by considering the relative distance to the 
maximum expected profit for both firms:

$$
\begin{gathered}
\Delta^{A}\left(y_{\alpha}, y_{\beta}\right)=\max \left(\left\{E^{A}\left(y_{i}, y_{\beta}\right)\right\}_{y_{i} \in Y}\right)-E^{A}\left(y_{\alpha}, y_{\beta}\right) \\
\lambda=\underset{i}{\arg \min }\left(\left\{\Delta^{A}\left(y_{i}, y_{\beta}\right)+\Delta^{B}\left(y_{i}, y_{\beta}\right)\right\}_{y_{i} \in Y}\right)
\end{gathered}
$$

Experiments. Participants were recruited using the Amazon Mechanical Turk (AMT) platform. AMT is an online crowdsourcing service where anonymous online workers complete webbased tasks in exchange for monetary compensation. It was noted that responses from AMT participants were at least as reliable as those obtained in laboratories (Buhrmester et al., 2011; Amir et al., 2012). In addition, AMT participants exhibit similar judgment and decision biases such as framing effects, conjunction fallacy, or outcome bias (Paolacci et al., 2010). The ethics approval for this project was provided by the Ecole Normale Supérieure as per the school's guidelines. In line with ethical guidelines, all participants provided informed consent before proceeding to the experiment. Participants also had to fill in a survey asking their age, nationality, and gender. Monetary compensation of one dollar was offered to each participant, with a bonus proportional to their score. In average, participants received a compensation of $\$ 2.64( \pm 0.58$ SD). Participants were paired inside a dedicated virtual room and each pair went through one of the four treatments. The four treatments correspond to the combination of two factors: consumers' view radius $(r)$ and the display of the opponent's profit $(s)$. The consumers' view radius that was either low $(r=0.25)$ or high $(r=0.50)$ and the opponent's profit was either hidden $(s=0)$ or displayed $(s=1)$. For all the rounds, we used the same parameters as for simulations except that we maintained constant the initial locations of firms: one of the two firms was placed at one of the extrema of the segment, the other firm at the other extrema. Their initial price was set to 5 . The subject playing first was randomly selected. The number of rooms (with two subjects each) for each condition is: $\left(r=0.25, \quad s=1, \quad n_{\mathrm{eco}}=26\right), \quad(r=0.25, \quad s=1$, $\left.n_{\text {eco }}=30\right),\left(r=0.50, s=1, n_{\text {eco }}=26\right),\left(r=0.50, s=1, n_{\text {eco }}=26\right)$. Additional information is provided in the supplementary section.

Analysis. Only data obtained from subjects that have fully completed the experimental procedure has been used for analysis. Among the 410 subjects that signed up to the platform, 222 subjects went through all the process (see Supplementary for more information). The reasons why a subject may not have completed the procedure are (i) the impossibility to match him with another subject, (ii) quit before the end, (iii) a technical problem (i.e., poor computer performances). The sample of subjects we used for analysis matches the demographic characteristics of AMT (Ipeirotis, 2010). Regarding the composition of the participants, we noticed a quasi-gender parity (women represented $54.1 \%$ and men $45.9 \%$ ). The average age was $34.75 \pm$ 9.54. We counted a dozen nationalities, the most common being American (75.78\%) with a large majority, and, to a lesser extent, Indian (11.71\%).

We drew a three-dimensional profile for each subject. Each dimension corresponds to a particular decision rule (PM, DM, TC). The score associated with each dimension assesses the extent to which a subject behaves accordingly to what a specific decision rule implies to do.

Let be $v_{H}^{A}\left(y_{\alpha}, y_{\beta}\right)$ be the value of the move $y_{\alpha}$ relatively to the decision rule $H \in\{\mathrm{PM}, \mathrm{DM}, \mathrm{TC}\}$ (respectively, for PM, DM,
TC) at time $t \in\left[1, n_{\text {turn }}\right]$ :

$$
\begin{aligned}
& v_{\mathrm{PM}}^{A}\left(y_{\alpha}, y_{\beta}\right)= \\
& \left\{\begin{array}{cl}
\frac{E^{A}\left(y_{\alpha}, y_{\beta}\right)}{\max \left(\left\{E^{A}\left(y_{i}, y_{\beta}\right)\right\}_{y_{i} \in Y}\right)} & \text { if } \max \left(\left\{E^{A}\left(y_{i}, y_{\beta}\right)\right\}_{y_{i} \in Y}\right)>0, \\
& 1 \text { otherwise. }
\end{array}\right. \\
& \left\{\begin{array}{cc}
v_{\mathrm{DM}}^{A}\left(y_{\alpha}, y_{\beta}\right)= \\
\frac{E^{A}\left(y_{\alpha}, y_{\beta}\right)-E^{B}\left(y_{\alpha}, y_{\beta}\right)}{\max \left(\left\{E^{A}\left(y_{i}, y_{\beta}\right)-E^{B}\left(y_{i}, y_{\beta}\right)\right\}_{y \in Y}\right)} & \text { if } \max \left(\left\{E^{A}\left(y_{i}, y_{\beta}\right)-E^{B}\left(y_{i}, y_{\beta}\right)\right\}_{y i \in Y}\right)>0, \\
& 1 \text { otherwise. }
\end{array}\right. \\
& \begin{array}{c}
v_{\mathrm{TC}}^{A}\left(y_{\alpha}, y_{\beta}\right)= \\
\left\{\begin{array}{c}
\frac{\min \left(\left\{\Delta^{A}\left(y_{i}, y_{\beta}\right)+\Delta^{B}\left(y_{i}, y_{\beta}\right)\right\}_{y_{i} \in Y}\right)}{\Delta^{A}\left(y_{\alpha}, y_{\beta}\right)+\Delta^{B}\left(y_{\alpha}, y_{\beta}\right)} \\
\text { if } \Delta^{A}\left(y_{\alpha}, y_{\beta}\right)+\Delta^{B}\left(y_{\alpha}, y_{\beta}\right)>0, \\
1 \text { otherwise. }
\end{array}\right.
\end{array}
\end{aligned}
$$

For convenience, we did not include the variable $t$ in the definition of the $\mathrm{v}$ functions. So, let's assume a function $f$ such as:

$$
f_{H}(i, t)=v_{H}^{i}\left(y_{\alpha}, y_{\beta}\right) \text { for time } t
$$

The score for a subject $i$ and for a decision rule $t$ is simply the average value of $f$ over time:

$$
s_{H}(i)=\frac{1}{n_{\mathrm{turn}}} \sum_{t}^{n_{\mathrm{turn}}} f_{H}(i, t)
$$

For the analysis, we pooled the individual scores by experimental condition. As we did not expect a normal distribution of the data due to clustering effects at the boundaries of our scales (i.e., price), assessment of statistic relevancy of our observations has been made with Mann-Whitney's $U$-ranking test, applying Bonferroni's corrections for multiple comparisons. We set the significance threshold at $1 \%$.

\section{Results}

Simulations. In order to test our hypothesis regarding the influence of the information level of consumer (measured by his view radius) on the differentiation of the two firms, we ran 1000 simulations using a random view radius between 0 and 1 and tested three different decision rules for the firms, namely PM, DM, and TC. For each of these simulations, we measured the mean distance between the two firms, which is the distance separating the two firms averaged over the last third of the 100 turns (i.e., the last 33 turns). We report in Fig. 2 all these distances on the $y$-axis and the corresponding view radius on the $x$-axis. Minimal differentiation corresponds to a mean distance of 0 firms being placed at the center of the linear city while maximal differentiation corresponds to a mean distance of 0.5 one firm being placed on the first quarter of the linear city and the other one at the last quarter.

The high dispersion of the points when $r$ is close to 0 or near 1 can be explained by the fact that the firm location has almost no impact on the firm profits. If the value of $r$ is close to zero, the consumers are almost blind in the sense that their view radius is so narrow that except if the competitor is very close, each firm would sell its product to only a few consumers, regardless of its position. If $r$ is close to 1 , the visual field of the consumer is so broad that it will see both firms and these firms will always compete. For such extreme values of $r$, the mean distance 
A

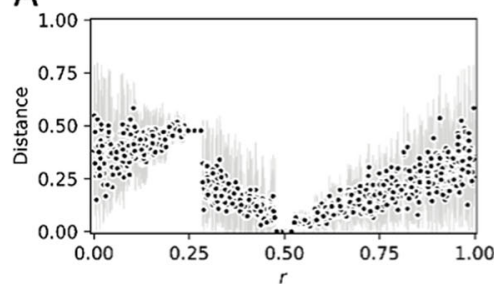

C

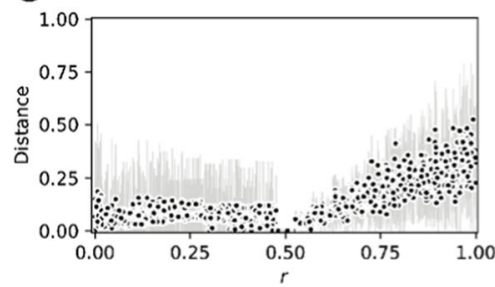

$\mathrm{E}$

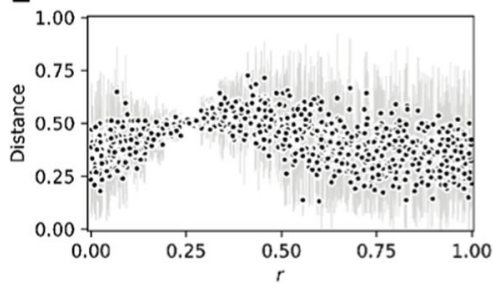

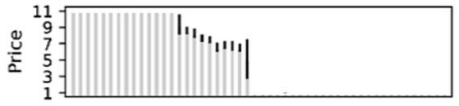
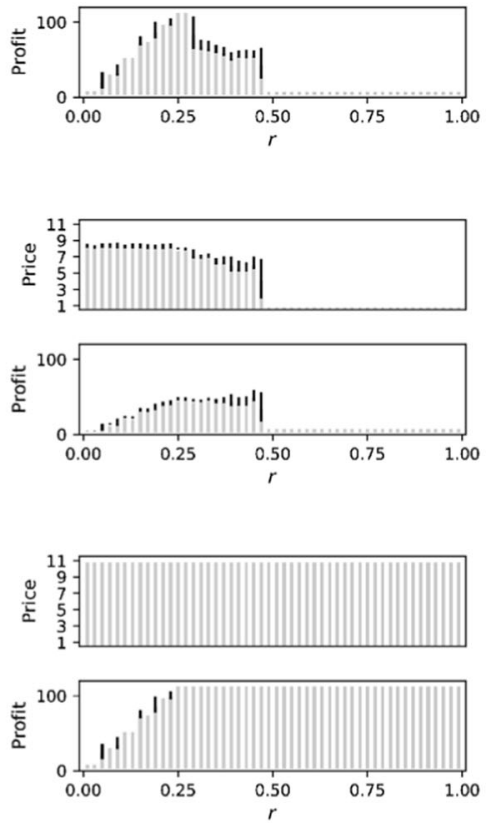

$\mathrm{B}$
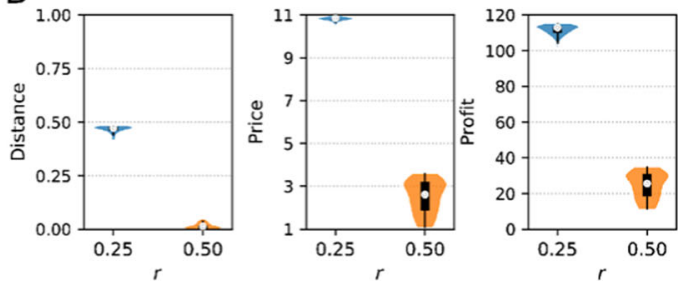

D
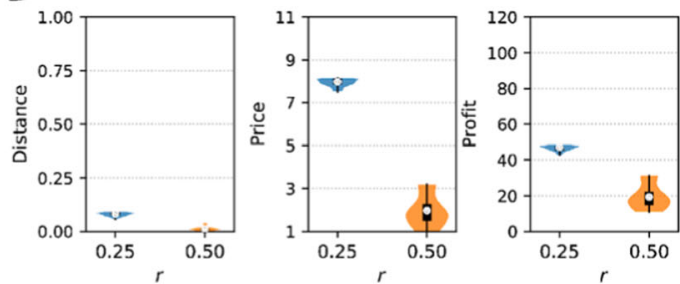

$\mathrm{F}$
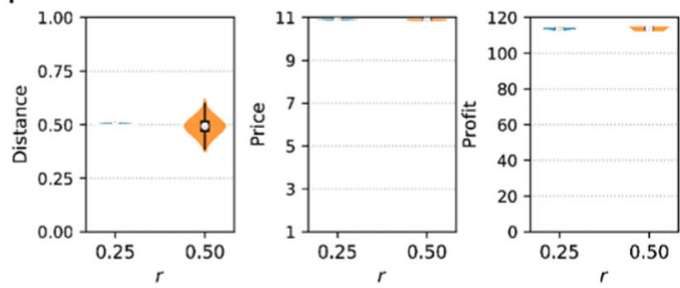

Fig. 2 Simulation results. For each of the three decision rules (profit maximization [PM], difference maximization [DM], tacit collusion [TC]),

1000 simulations were run with a random (uniform) view radius for the consumers. The distance between the two firms, the profit and the price as a function of the consumers' view radius is displayed in $\mathbf{a}(\mathrm{PM}), \mathbf{c}(\mathrm{DM})$, and $\mathbf{e}(\mathrm{TC})$. Each dot corresponds to the mean distance that has been observed between the two firms during a single simulation and vertical bars indicate the standard deviation. Mean prices and profits are reported on the right using gray bars and the standard deviation in black. For each of the three decision rules (PM, DM, TC) and for low- and high-view radius $(r=0.25, r=0.50), 25$ additional simulations were run and observed distance, price, and profit are displayed on the right $(\mathbf{b}, \mathbf{d}, \mathbf{f})$ in order to compare them to the experimental results

observed is close to 0.33 , which corresponds to the mean distance observed for random moves. As each consumer sees only its own position or sees both firms, it is indeed expected that the firms randomly choose their location, which corresponds to a mean distance of 0.33 .

We can observe in Fig. 2a that for the PM decision rule, a view radius of 0.50 corresponds to the minimal differentiation principle where the two firms compete to occupy the central position because this is the unique position that gives access to all the consumers (all consumers are potential consumers for the firm positioned at the center). It thus makes sense for the two firms to compete around this position and to try to get a maximum number of consumers in order to maximize their profit. Because of this competition, the mean price for both firms is very low and leads to moderate profits. When the view radius is reduced to 0.25 , the mean distance between the two firms is maximal (0.5). This specific radius corresponds to a case where there is a possibility of local markets as shown on Fig. 1. The two ends of the plateau when $r=0.25$ represent a compromise between competition and a lesser number of consumers, but those consumers are captive for each firm. This allows both firms to set higher prices and to maximize their profits. When the DM decision rule is used (see Fig. 2c), firms tend to minimally differentiate, the proximity forcing them to reduce their prices and hence, greatly reducing their profits compared to what happens with firms using a PM decision rule. As one would expect, when firms try to optimize at the same time their profit and their opponent's profit (TC decision rule), firms tend to maximally differentiate and establish local monopolies (see Fig. 2e).
Focusing our attention on the specific cases where $r=0.25$ or $r=0.50$ (Fig. $2 \mathrm{~b}, \mathrm{~d}$ and $\mathrm{f}$ ), one can notice quite different situations in terms of distance, price and profits for the three policies respectively. For $r=0.25$, the principle of maximal differentiation applies for PM and TC decision rules, leading to maximal prices and profits. This is not true for the DM decision rule where the principle of minimal differentiation seems to apply, leading to moderate prices and profits. For $r=0.50$, the situation is different and both the PM and DM decision rules lead to a minimal differentiation of the two firms with low prices and profits. Only the tacit collusion decision rule (TC) allows for an implicit equal share of the market, with highest prices and profits. Together, these three decision rules allow to give account on minimum or maximum differentiation in the two specific cases of low and high level of information available to the consumers.

Experiments. When considering the effect of the view radius of consumers on the mean distances, prices and profits (each of these measures being an average for each pair of subjects, in such a manner that each observation accounts for a two subjects couple), experimental results are very similar to the results of simulations when the PM decision rule is used, and this, independently of whether the opponent's profit is visible or not. Indeed, a large view radius induces a minimal differentiation effect where the two firms are led to a fierce competition around the central location, subsequently decreasing their prices and profits. Conversely, when consumers dispose of a narrow view radius, firms tend to exploit this disability by locating at the endpoints of the segment. 
More precisely, the median distance is greater when $r=0.25$ than when $r=0.50$ (when $s=0, u=81.5, p<0.001, n=59$; when $s=1, u=33.0, p<0.001, n=52)$. The same applies for the prices (when $s=0, u=128.5, p<0.001, n=59$; when $s=1, u=30.5, p$ $<0.001, n=52$ ) and for the profits (when $s=0, u=178.0, p<$ $0.001, n=59$; when $s=1, u=131.5, p<0.001, n=52$ ).

The display of the opponent's profit $(s=1)$ has a limited effect on the general shape of the data relatively to distance, price, and profit. It has an effect on distance only when $r=0.25$ (when $r=0.25, u=191.0, u=191.0, p=0.007, n=56$; when $r=0.50$, $u=283.0, p=0.686, n=55$ ) and no effect on price (when $r=0.25, u=321.0, p=1.000, n=56$; when $r=0.50, u=311.0$, $p=1.000, n=55$ ) and profit (when $r=0.25, u=252.0$, $p=0.143, n=56$; when $r=0.50, u=296.5, p=1.000, n=55)$.

A table summarizing the results is available in the supplementary section (see Table S4).

Although the general shape of data is close to what has been observed with simulations using the PM decision rule, the dispersion of results is much more spread out and we assume this scattering of the data can be attributed to inter-individual differences. In order to study this inter-individual variability, we computed three individual scores for each subject, assessing the compatibility of their behavior for each time step of the experiment with the use of (i) a PM decision rule, (ii) a DM decision rule, (iii) a TC decision rule. Distribution by experimental condition of PM, DM, and TC scores are shown in Fig. 3b. A matrix correlation of the scores by experimental condition has also been computed (see Fig. 3c).

Considering the effect of the field of view on individual scoring, the results indicate that the DM scores are higher in condition of high information while PM scores are lower. The variation of the view radius has no significant impact on TC scores. More precisely, considering the effect of field of view on individual scoring and comparing when $r=0.50$ to when $r=0.25$, for both value of $s$, we observe that the DM score are significantly higher (when $s=0, u=488.0, p<0.001, n=118$; when $s=1, u=460.0$, $p<0.001, n=104)$ and the PM scores are significantly lower (when $s=0, u=1048.0, p<0.001, n=118$; when $s=1$, $u=861.0, p<0.001, n=104)$. Still when $r=0.50$ compared to when $r=0.25$, the TC scores are significantly lower, but only when $s=1$ (when $s=0, u=1452.5, p=0.730, n=118$; when $s=1, u=461.5, p<0.001, n=104)$.

Considering the effect of the display of the opponent's score, the results indicate that if the opponent's score is displayed, the DM scores are higher in condition of low information. In contrary, it has no significant impact on PM and TC scoring. More precisely, considering the effect of opponent's profit displaying on individual scoring (i.e., when $s=1$ compared to when $s=0$ ), the DM score are significantly higher only when $r=0.25$ (when $r=0.25, u=733.0, p<0.001, n=112$; when $r=0.50, u=1031.0, p=0.026, n=110)$, while the PM scores are not statistically different (when $r=0.25, u=1248.5, p=0.413$, $n=112$; when $r=0.50, u=1438.5, p=1.000, n=110)$, neither are the TC scores (when $r=0.25, u=1204.5, p=0.227, n=112$; when $r=0.50, u=1376.5, p=0.216, n=110)$. A table summarizing the results is available in the supplementary section (see Table S5).

Also, similarly to the results obtained by simulation, a radius value of 0.25 allows to discriminate the use of a PM decision rule from a TC decision rule, and a radius value of 0.50 allows to discriminate the use of a PM decision rule from a DM decision rule. This is especially noticeable when looking at the distribution of the scores (Fig. 3b) but also when looking at the correlation matrix (Fig. 3c). When $r=0.25$, a subject who has a high score in PM would likely to have a high score in TC but a low score in $\mathrm{DM}$, while when $r=0.50$, a subject who has a high score in PM would likely have a high score in DM but a low score in TC. Hence, when trying to discriminate different decision rules, a condition of low information $(r=0.25)$ allows to distinguish a PM from a DM decision rule, but not from a TC decision rule. Conversely, in a condition of high information $(r=0.50)$, a DM is indistinguishable from a PM decision rule, but a TC decision rule is. In other words, a DM decision rule could be interpreted as a decision rule revealed solely in a condition of low information, while a TC decision rule could be interpreted as an adaptive decision rule in condition of high information.

If we now look more closely at individual behaviors, it is striking to see that when subjects competing together have been identified both as users of a specific decision rule (i.e., obtained a high score toward PM, DM, or TC), the dynamics of their playing is very similar to the corresponding simulation. With $r=0.25$, subjects using PM decision rule tend to position themselves at the first and third quarters of the segment and both set a high selling price (see Fig. 4a). However, when $r=0.50$, subjects position themselves at the center and immediately lower their price (see Fig. 4b) even though they are less inclined to do so compared to simulated firms using the corresponding decision rule. They are actually trying to regularly increase their price. The situation is a bit different for subjects using a DM decision rule when $r=0.25$ (see Fig. 4c). In that case, the positions of subjects oscillate around the center accompanied with an increase and decrease in prices, indicating a will to capture the market of their opponent. When $r=0.50$, both simulated firms and human subjects using a TC decision rule set their prices at their maximum but the dynamics are not exactly the same (see Fig. 4f). Subjects positioned themselves further apart, and this increase of the distance can be assumed to be due to an intent from the subjects to communicate their goodwill to their opponent.

\section{Discussion}

The principle of minimal differentiation as exposed in the seminal paper of Hotelling (1929) did not reach consensus in the abundant subsequent literature. Once some restrictive assumptions of the initial model are relaxed (for instance, number of firms, spatial structure, or cost structure), it has been shown that the principle of minimal differentiation can be invalidated and that the antagonistic principle of maximal differentiation could apply (d'Aspremont et al., 1979; Cremer et al., 1991; Economides, 1993; Brenner, 2005). In addition of these theoretical results, several experimental studies show that by manipulating either the communication between firms (Kruse et al., 2000) or by manipulating the time structure (Kephart and Friedman, 2015) - what also indirectly impacted the ability of the firms to communicate about their intentions - it was possible to induce either a minimal or a maximal differentiation between the firms. Similarly to Kruse et al. (2000) and Kephart and Friedman (2015), and despite the robustness of the minimal differentiation principle highlighted by the experimental results of Huck et al. (2002) and BarredaTarrazona et al. (2011), our results report both phenomena: simulations and experiments allowed us to demonstrate that the consumers' amount of information affects the differentiation of firms with respect to their decision-making strategies. We isolated incentives supporting either a geographic concentration and a fierce price competition resulting in drastic reduction of profits, or a maximal differentiation inducing a softening of the price competition and thereby a large increase in firms' profits. However, our results also show that the principle of maximal differentiation may be systemic and cannot be uniquely attributed to the deliberate use of a cooperative strategy on the part of firms (as in Kruse et al., 2000), or to TC (as in Kephart and Friedman, 2015). 
A
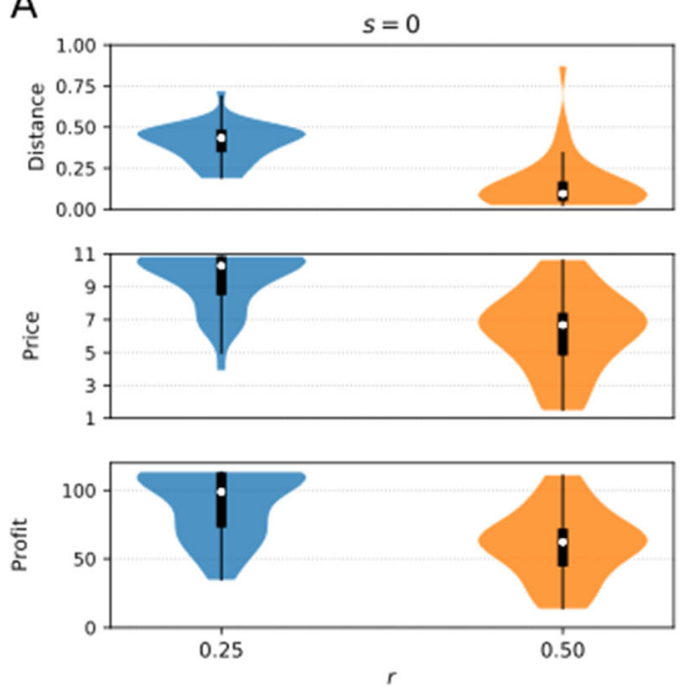

B
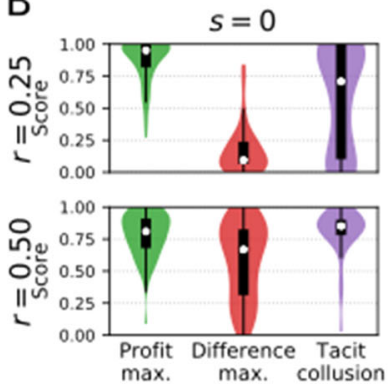
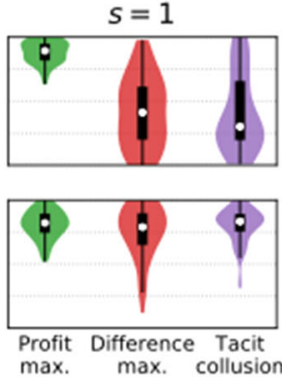
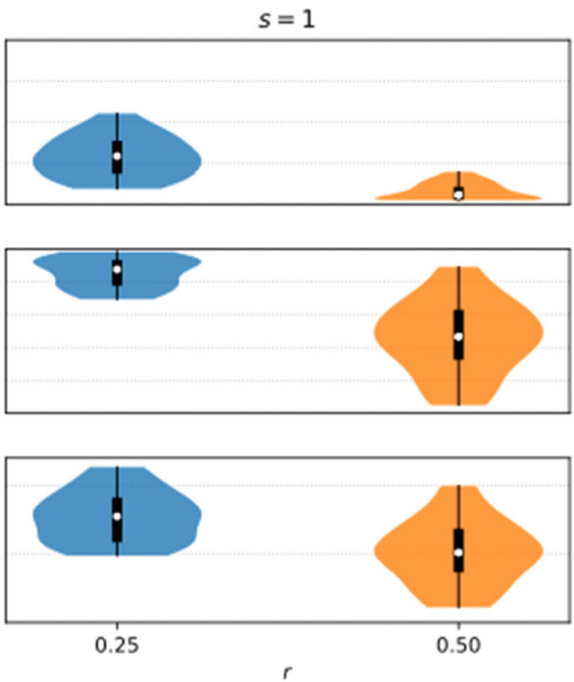

C
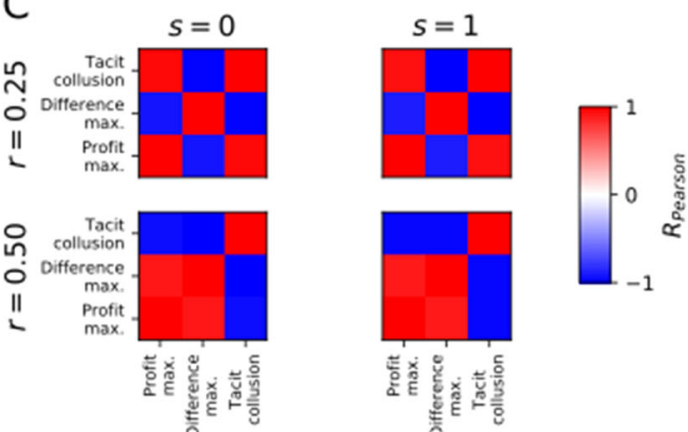

Fig. 3 Experimental results. a Combined effect of the consumers' view radius and the display of the opponent's score on distance, price, and profit. The white dots indicate the median, the thick black bars indicate the IQR. The extrema of the thin bars indicate the lower and upper adjacent values. The colored areas give an indication of the shape of the data distribution. $\mathbf{b}$ Mean scores of profit maximization (PM), difference maximization (DM), and tacit collusion (TC) by experimental condition. The white dots indicate the median, the thick black bars indicate the IQR. The extrema of the thin bars indicate the lower and upper adjacent values. The colored areas give an indication of the shape of the data distribution. c Score correlation matrix. Blue color indicates a strong negative correlation and red color a strong positive correlation

Indeed, when consumers have only access to a low level of information, the occurrence of maximal differentiation in experimental results can in turn be interpreted as an adaptation to these consumers' limited access to information. In that circumstance, firms using a PM decision rule formed local monopolies without any willingness to cooperate with the other firm. This supports and provides a possible rationale to d'Aspremont et al.'s final open remark in their fundamental reexamination of Hotelling's model (1929), according to which, contra Hotelling, one should intuitively expect differentiation to be a distinctive feature of oligopolistic competition. Oligopolists should indeed be better off by dividing the markets into submarkets over which they each exert quasi-monopolistic control. Our results actually demonstrate that limited access to information, on the part of consumers, can be an underlying factor and a prevailing one in actual competitive markets that induces a non-competitive behavior from which firms, without prior explicit collusion, can take advantage of the situation and establish local monopolies, which are detrimental to consumers. Our results show that only the use of a profit DM decision rule precludes the formation of such local monopolies.

Besides, the use of these decision rules allowed us to emphasize heterogeneous behaviors. Thinking of these various behaviors in terms of deviation from a rational behavior understood as the maximization of a unique utility function would have prevented us from making sense of this heterogeneity. In order to define our decision rules, we measured whether our subjects looked for maximizing their own profit, whether they aimed at maximizing the difference of profits with their opponents, or finally whether they tried to create a TC. The PM decision rule appears to be a good predictor of the firms' aggregated behavior, while the other decision rules offer an opportunity to account for less expected behaviors.

The use of a DM decision rule indeed supported a fierce competition when informational structure opened the possibility of quasi-monopolies. The use of this decision rule could be explained by the presence of an underlying anchoring bias (Tversky and Kahneman, 1974): as it is difficult to evaluate the success of a move per se, a move is considered efficient if it leads to beer profits than its opponent. In other words, firms' strategy evaluation relies on comparisons to a given point instead of an evaluation in absolute terms. This could explain why this decision rule has been promoted by the display of the opponent score. The use of such decision rule could also be due to an underlying zerosum bias (Meegan, 2010; Różycka-Tran et al.,): considering wrongfully that a greater profit for its opponent is necessarily a profit loss for itself, a firm could decide to make its choice only considering the profit difference.

While DM decision rules are precluded under certain conditions the formation of monopolies, the use of a TC decision rule 
A
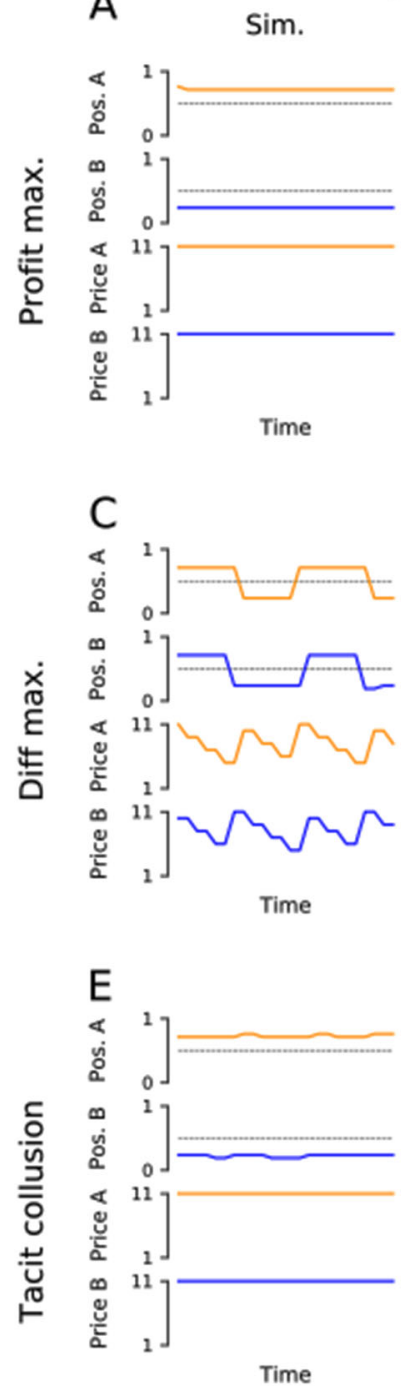

$r=0.25$

Exp.
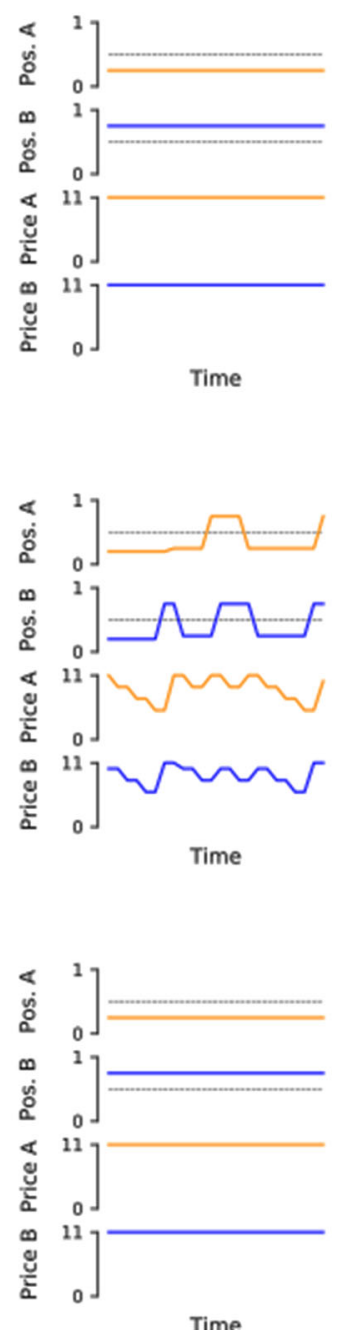

B
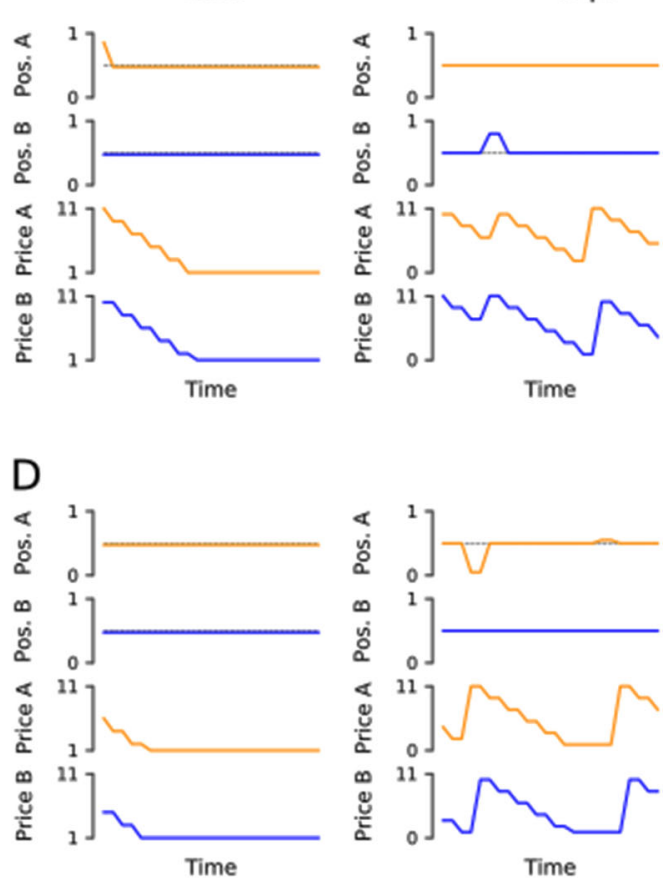

$\mathrm{F}$

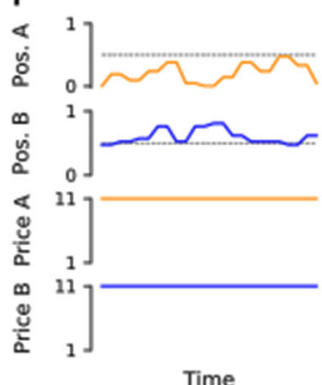

$r=0.50$

Exp.
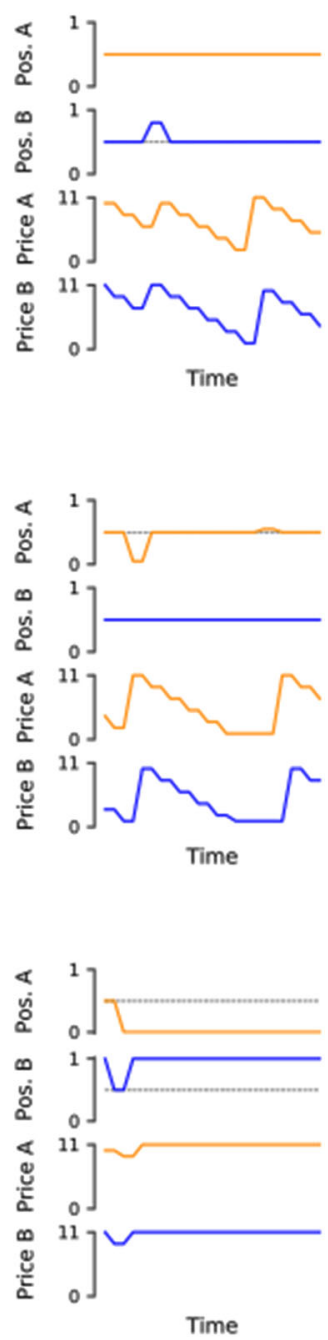

Fig. 4 Analysis of dynamics. Comparison of the dynamics between artificial firms and human controlled firms. Each figure presents the evolution of positions and prices of two firms in competition (orange: Firm A, blue: Firm B; data for artificial firms comes from a single simulation that serves as an example of a typical behavior). a Left: artificial firms using a profit maximization strategy; right: two participants with a high score in profit maximization; $r=0.25$. b Same as in subfigure $\mathbf{a}$ but with $r=0.50$. c Left: two firms using a difference maximization (DM) decision rule; two participants with a high score in $\mathrm{DM} ; r=0.25$. d Same as in subfigure $\mathbf{c}$ but with $r=0.50$. e Left: two firms using a tacit collusion (TC) decision rule; right: two participants with a high score in TC; $r=0.25$. f Same as in subfigure e but with $r=0.50$

allowed to relax price competition when information structure was promoting it. As a means to avoid the drawbacks of a competition situation leading to lower profits, the use of such decision rule could be explained by the search for a Pareto optimality (Pareto, 1964) that is to say following the strategies that lead to a distribution of profits such as no firm could earn more, otherwise it would be at the expense of the other. It could also be interpreted as deliberate attempts to emit signals in order to relax competition in a situation where the communication technology needed to lead it rationally is lacking.

Another consideration that is raised by our study is that the consequences of using such decision rules can differ from Nash predictions applied to a basic Hotelling's model under full information: for instance, the use of a TC decision rule under full information leads to maximally differentiate while minimal differentiation would be expected. However, it is now a well-trodden theme that decision rules can be interpreted in terms of their adaptive rationality (Gigerenzer and Reinhard, 2001). As long as a chosen decision rule improves the outcome of the game and corresponds to relatively stable observable spatial patterns, we can speak of a specific form of rationality arising under the imposed informational constraint. Work by Sutton (1997), applied to the Hotelling's model, explores such an equilibrium notion and weak rationality requirement, based in his case on a single decision rule, which is to seize an opportunity when it presents itself. It would take a further study to understand how the TC decision rule highlighted here indeed constitutes an adaptive rational behavior to informational constraints, either exerted on consumers by means of the availability of information, or exerted on firms by means of their disability to communicate.

For the purpose of our study, we considered a model with a basic architecture. For instance, we used a homogeneous view radius for consumers in our model, mainly for two reasons: (i) it may have been more difficult for the subjects to identify consumers view radius and to adapt their behavior in consequence, (ii) diminish the potential variability of our data. Besides, from a more theoretical point of view, it is equivalent to consider constant radius across the population of consumers as reciprocally 
implementing the idea of a limited sphere of influence of the firms. Given this limited amount of vision around consumers or sphere of influence of firms (in the sense, then, of being visible by consumers) it also motivates firms to try to change their location. That being said, It might be relevant to implement heterogeneous view radius in a further study, in order to test the robustness of our results established in a homogeneous setting.

Another implication of our implementation of information is that consumers may be unaware of one of the two options. The unawareness of one of the two options is an abstraction for a consumer that is not willing to inform himself. For instance, we can mention emergency situations (keys lost, car break) where the first solution is picked without consideration of other options. This type of partial attention or restriction to a "consideration set" can also be seen as reflecting a form of incomplete preference relation on the part of the consumers, which has been modeled in different terms in the literature: top options (Rubinstein and Salant, 2011) or consideration sets (Lleras et al., 2017).

Regarding the structure of firm decision-making, we think that a turn-based strategy is more appropriate as it is more likely that a human subject embodying a firm chooses a strategy in reaction to a change of strategy from its competitor. When dealing with rational agents, simultaneous decision-making is possible in the sense that they dispose of full information and unbounded computational abilities, providing them the means to compute the equilibrium and to play accordingly. As we set-up a human subject experiment, we were expecting to deal with non-fully rational agents that are unable to do so. Coordination on price or location policies, leading to a typical situation of maximal/minimal differentiation seems unlikely or at least much more difficult in this configuration. Indeed, pure rationality models such as required to deal with normal forms or extensive forms in experimental game-theory predict behavior to a lesser extent that taking account the incremental feedback of players in repeated sequential situations (Roth and Erev, 1995). We anticipated that subjects will make use of decision rules, leading to more or less stable situations-these decision rules play also the rules of learning heuristics. Then, designing a turn-based game constituted for us a way to facilitate the occurrence of such situations.

From a broader perspective, our results demonstrate interaction effects between consumers and firms' cognitions, that can deeply impact market dynamics. It, therefore, creates an incentive to think that duopoly regulation should incorporate insights from incomplete markets due to agents limited cognitive abilities. However, most of the focus has been put in behavioral industrial organization to the irrationalities of clients rather than firms. We donot consider our firms irrational either but as constrained to find decision rules in response to their own perception of the consumers' limited information about themselves. From a theoretical point of view, we are not the first to envision such a problem (Spiegler, 2006). However, the decision rules we stylize and simulate can definitely provide incentives to a more behaviorally oriented approach to duopoly regulation.

\section{Code and data availability}

Simulations were implemented using Python and the Python scientific stack (Jones et al., 2001; van der Walt et al., 2011; Hunter, 2007). The code is available at https:/github.com/ AurelienNioche/SpatialCompetition.

The software used for the experimental part of the study is based on a client/server architecture. The client part was developed using the Unity game engine, hosted on a dedicated server and ran in the subjects' web browser using WebGL API. The code and the assets are available at https://github.com/AurelienNioche/
DuopolyAssets. The experiment server was hosted on a dedicated server and developed using the Django framework. The code of the server part is available at https:/github.com/AurelienNioche/ DuopolyDjango.

The analysis program is available at https://github.com/ AurelienNioche/DuopolyAnalysis. Figures 3 and 4 were produced using raw data that are available at https://github.com/ AurelienNioche/DuopolyAnalysis.

Received: 26 September 2018 Accepted: 28 February 2019

Published online: 26 March 2019

\section{References}

Amir O, David GR, Yaakov KG (2012) Economic games on the internet: The effect of \$1 stakes. PLoS ONE 7(2):e31461. https://doi.org/10.1371/journal.pone.0031461

Barreda-Tarrazona I, Aurora G-G, Nikolaos G, Joaquín A-F, Agustín G-S (2011) An experiment on spatial competition with endogenous pricing. Int J Ind Organ 29(1):74-83. https://doi.org/10.1016/j.ijindorg.2010.02.001

Brenner S (2005) Hotelling games with three, four, and more players. J Reg Sci 45 (4):851-64. https://doi.org/10.1111/j.0022-4146.2005.00395.x

Brown S (1989) Harold hotelling and the principle of minimum differentiation. Progress Human Geogr 13(4):471-93. https://doi.org/10.1177/03091325890 1300401

Buhrmester M, Tracy K, Samuel DG (2011) Amazons mechanical turk. Perspect Psychol Sci 6(1):3-5. https://doi.org/10.1177/1745691610393980

Cremer H, Maurice M, Jacques-François T (1991) Mixed oligopoly with differentiated products. Int J Ind Organ 9(1):43-53. https://doi.org/10.1016/01677187(91)90004-5

d'Aspremont C, Jaskold Gabszewicz J, Thisse J-F (1979) On hotellings "stability in competition”. Econometrica 47(5):1145. https://doi.org/10.2307/1911955

Dudey M (1990) Competition by choice: The effect of consumer search on firm location decisions. Am Econ Rev 80(5):1092-1104. https://ideas.repec.org/a/ aea/aecrev/v80y1990i5p1092-1104.html

Eaton BC, Richard GL (1975) The principle of minimum differentiation reconsidered: Some new developments in the theory of spatial competition. Rev Econ Stud 42(1):27. https://doi.org/10.2307/2296817

Economides N (1993) Hotelling's "main street" with more than two competitors. J Reg Sci 33(3):303-19. https://doi.org/10.1111/j.1467-9787.1993.tb00228.x

Gigerenzer G, Reinhard S (eds.) (2001). Bounded rationality: The adaptive toolbox. Cambridge, MA: MIT Press. https://mitpress.mit.edu/books/bounded-rationality

Hotelling H (1929) Stability in competition. Econ J 39(153):41. https://doi.org/ $10.2307 / 2224214$

Huck S, Wieland M, Nicolaas JV (2002) The east end, the west end, and king'cross: On clustering in the four-player hotelling game. Econ Inq 40(2):231-40. https://doi.org/10.1093/ei/40.2.231

Hunter JD (2007) Matplotlib: A 2D graphics environment. Comput Sci Eng 9 (3):90-95. https://doi.org/10.1109/MCSE.2007.55

Ipeirotis PG (2010) Demographics of mechanical turk. Ce-DER-10-01. New York University, New York, NY, http://www.ipeirotis.com/wp-content/uploads/ 2012/02/CeDER-10-01.pdf

Irmen A, Jacques-François T (1998) Competition in multi-characteristics spaces: Hotelling was almost right. J Econ Theory 78(1):76-102. https://doi.org/ 10.1006/jeth.1997.2348

Jones E, Travis O, Pearu P (2001) SciPy: Open source scientific tools for python. http://www.scipy.org

Kahneman D (2003) A perspective on judgment and choice: Mapping bounded rationality. Am Psychol 58(9):697-720. https://doi.org/10.1037/0003-066x.58.9. 697

Kephart C, Friedman D (2015) Hotelling revisits the lab: Equilibration in continuous and discrete time. J Econ Sci Assoc 1(2):132-45. https://doi.org/ $10.1007 / \mathrm{s} 40881-015-0009-\mathrm{z}$

Kruse JB, David JS (2000) Location, cooperation and communication: an experimental examination. Int J Ind Organ 18(1):59-80. https://doi.org/10.1016/ s0167-7187(99)00034-X

Lleras JS, Masatlioglu Y, Nakajima D, Ozbay EY (2017) When more is less: Limited consideration. J Econ Theory 170:70-85. https://doi.org/10.1016/j.jet.2017.04.004

Loertscher S, Muehlheusser G (2011) Sequential location games. RAND J Econ 42 (4):639-63. https://doi.org/10.1111/j.1756-2171.2011.00148.x

Meegan DV (2010) Zero-sum bias: perceived competition despite unlimited resources. Front Psychol 1:191. https://doi.org/10.3389/fpsyg.2010.00191

Paolacci G, Jesse C, Panagiotis GI (2010) Running experiments on amazon mechanical turk. Judgm Decis Mak 5(5):411-19 https://EconPapers.repec. org/RePEc:jdm:journl:v:5:y:2010:i:5:p:411-419 
Pareto V (1964). Cours d'économie Politique. vol 1. Librairie Droz. https://www. cairn.info/cours-d-economie-politique-tomes-1-et-2--9782600040143.htm

Prescott EC, Visscher M (1977) Sequential location among firms with foresight. Bell J Econ 8(2):378. https://doi.org/10.2307/3003293

Różycka-Tran J, Paweł B, Bogdan W (2015) Belief in a zero-sum game as a social axiom: A 37-nation study. J Cross-Cult Psychol 46(4):525-48. https://doi.org/ $10.1177 / 0022022115572226$

Roth AE, Erev I (1995) Learning in extensive-form games: Experimental data and simple dynamic models in the intermediate term. Games Econ Behav 8 (1):164-212. https://doi.org/10.1016/S0899-8256(05)80020-X

Rothschild A (1947) Price theory and oligopoly. Econ J 57(227):299-320. https:// doi.org/10.2307/2225674

Rubinstein A (1991) Comments on the interpretation of game theory. Econometrica 59(4):909-924. https://doi.org/10.2307/2938166

Rubinstein A, Salant Y (2011) Eliciting welfare preferences from behavioural data sets. Rev Econ Stud 79(1):375-387. https://doi.org/10.1093/restud/rdr024

Schultz C (2009) Transparency and product variety. Econ Lett 102(3):165-68. https://doi.org/10.1016/j.econlet.2008.12.008

Spiegler R (2006) Competition over agents with boundedly rational expectations. Theor Econ 1(2):207-231. https://econtheory.org/ojs/index.php/te/article/ viewArticle/20060207

Stahl K (1982) Differentiated products, consumer search, and locational oligopoly. J Ind Econ 31(1/2):97. https://doi.org/10.2307/2098007

Stigler GJ (1961) The economics of information. J Political Econ 69(3):213-25. https://doi.org/10.1086/258464

Sutton J (1997) One smart agent. RAND J Econ 28(4):605. https://doi.org/10.2307/ 2555778

Thaler R (1980) Toward a positive theory of consumer choice. J Econ Behav Organ 1(1):39-60. https://doi.org/10.1016/0167-2681(80)90051-7

Tversky A, Kahneman D (1974) Judgment under uncertainty: Heuristics and biases. Science 185(4157):1124-31. https://doi.org/10.1126/science.185.4157.1124

van der Walt S, Colbert SC, Gaël V (2011) The NumPy array: A structure for efficient numerical computation. Comput Sci Eng 13(2):22-30. https://doi. org/10.1109/mcse.2011.37

Webber MJ (1972) The impact of uncertainty upon location. MIT Press, Cambridge, MA, https://mitpress.mit.edu/books/impact-uncertainty-location

\section{Acknowledgements}

This work was supported by the Agence Nationale de la Recherche (ANR-16-CE380003). The funders had no role in study design, data collection, and interpretation, or the decision to submit the work for publication.

\section{Author contributions}

AN and BG wrote the code, performed the experiments and the data analysis; AN, BG, TB, NR, and SB-G designed the study and co-wrote the manuscript.

\section{Additional information}

Supplementary information: The online version of this article (https://doi.org/10.1057/ s41599-019-0241-x) contains supplementary material, which is available to authorized users.

Competing interests: The authors declare no competing interests.

Reprints and permission information is available online at http://www.nature.com/ reprints

Publisher's note: Springer Nature remains neutral with regard to jurisdictional claims in published maps and institutional affiliations.

(c) (i) Open Access This article is licensed under a Creative Commons Attribution 4.0 International License, which permits use, sharing, adaptation, distribution and reproduction in any medium or format, as long as you give appropriate credit to the original author(s) and the source, provide a link to the Creative Commons license, and indicate if changes were made. The images or other third party material in this article are included in the article's Creative Commons license, unless indicated otherwise in a credit line to the material. If material is not included in the article's Creative Commons license and your intended use is not permitted by statutory regulation or exceeds the permitted use, you will need to obtain permission directly from the copyright holder. To view a copy of this license, visit http://creativecommons.org/ licenses/by/4.0\%

(C) The Author(s) 2019 